\title{
RELATIONSHIPS BETWEEN ANTHROPOMETRIC PARAMETERS AND OVERARM THROW IN ELITE BEACH HANDBALL
}

original paper

() University School of Physical Education in Wroclaw

DOI: https://doi.org/10.5114/hm.2019.79394

\author{
JOSE M. JIMENEZ-OLMEDO ${ }^{1}$, ALFONSO PENICHET-TOMAS ${ }^{1}$, \\ MANUEL ORTEGA BECERRA ${ }^{2}$, BASILIO PUEO ${ }^{1}$, JOSE J. ESPINA-AGULLO ${ }^{1}$ \\ ${ }^{1}$ University of Alicante, Alicante, Spain \\ ${ }^{2}$ Pablo de Olavide University, Seville, Spain
}

\begin{abstract}
Purpose. Beach handball is a physically demanding sport. The aim of the study was to establish the relationships between anthropometric characteristics and overarm ball speed, a key performance factor.

Methods. Overall, 11 male players from the Spanish beach handball national team were recruited. Throw speed was assessed in 3 throw types: standing throw (ST), standing throw after two steps (RST), and jump throw with a run-up and self-spin (RSST). Pearson correlation was used to determine the correlation between variables. Magnitudes were evaluated qualitatively with magnitude-based inference, and the effects of anthropometric variables on the throw tests were analysed with stepwise multiple regression.

Results. Maximal throw speed was $25.64 \pm 4.14 \mathrm{~m} \cdot \mathrm{s}^{-1}$ for ST, $23.71 \pm 4.24 \mathrm{~m} \cdot \mathrm{s}^{-1}$ for RST, and $24.83 \pm 5.49 \mathrm{~m} \cdot \mathrm{s}^{-1}$ for RSST. Large positive correlations were found between throw speed and middle, ring, and little finger lengths (P4 polygon) $(r=0.66$ and 0.61 for ST and RSST, respectively). Very large $(r=0.77)$ and large $(r=0.68)$ correlations were observed between ST and RST, respectively, and elbow breadth. These results were likely positive for ST: P4 (76/24/0), elbow epicondyle distance (EED) (91/9/0); and for RST: EED (79/21/0); and possibly positive for RST: P4 (68/32/0). ST and RST were predicted by P4 in $44 \%$ and by combining P4 and finger span FS1 in 68\%.
\end{abstract}

Conclusions. ST and RST ball speed in beach handball was related to specific hand/elbow anthropometric characteristics which can be easily measured.

Key words: performance, handgrip, throw test, finger, ball speed, hand

\section{Introduction}

Ball-throwing velocity and accuracy can be considered as key factors in handball [1, 2]. Overarm throws are related with specific technical and tactical aspects [3], as well as with performance anthropometric characteristics of players [4]. The throwing ball velocity depends on upper and lower extremity power output capacity, with a direct relationship with dynamic strength, peak power, and peak ball velocity [5].

In the kinematic analysis field, the technical execution of overarm throwing movement is similar between genders. Maximal endpoint velocities of hand and wrist segments were larger for male than for female players [6]. It has been shown that $67 \%$ of ball velocity when it is released can be explained by the summation effects from velocity of elbow extension and internal rotation of shoulder. In turn, the shoulder contribution is influenced by arm position and by fatigue [7]. On the other hand, the throw type, either above or side, triggers different trunk flexions that affect ball release speed but throw accuracy remains unchanged [8]. Also, higher ball velocity is achieved in handball when players perform a run-up with pelvis and trunk movement, specifically with a delayed start to trunk flexion [9].

The relationship between specific anthropometric parameters and overarm throw performance has been studied extensively. Anthropometric profiles for specific player positions are known [10]. Such anthropometric

Correspondence address: José Manuel Jiménez-Olmedo, University of Alicante, Faculty of Education, Calle Aeroplano s/n, 03690 San Vicente del Raspeig, Alicante, Spain, e-mail: j.olmedo@ua.es

Received: July 24, 2018

Accepted for publication: October 24, 2018

Citation: Jimenez-Olmedo JM, Penichet-Tomas A, Becerra MO, Pueo B, Espina-Agullo JJ. Relationships between anthropometric parameters and overarm throw in elite beach handball. Hum Mov. 2019;20(2):16-24; https://doi.org/10.5114/hm.2019.79394. 
features as height with running speed, agility, and single leg horizontal jump distance can be used for talent detection in young players [11]. However, other studies suggest that anthropometric parameters are relatively poor predictors for scores on basic and specific motor ability tasks in handball, which requires agility, explosive strength of lower limbs, precision, movement speed, and handball-specific skills [12]. However, team handball players who are taller and have greater body weight perform higher ball release speeds in jump throw, and an increase in trunk flexion and rotation angular velocity improves ball release speed. The latter suggest that both anthropometrics and proper technique are needed for high overarm throw speeds [13].

Among all anthropometric features, hands and wrists are the key factors to reach the highest speeds in overarm throws. For this reason, some studies have focused on the relationship between specific hand measures and different throwing tests or handgrip strength $[14,15]$. It has also been suggested that hand dimensions could be a valid criterion for the selection of athletes [16].

Beach handball is a sport derived from team handball and has its specific differentiating characteristics. First, beach handball is played over a sandy field of game, with field dimensions, time per game period, as well as ball size, material, and dimensions differing from those in indoor handball. Also, different indoor and outdoor weather conditions, like humidity, temperature, and wind, play a role in players' performance [17]. As a result, these characteristics may be conditional factors influencing overarm throw in beach handball.

However, despite the existence of a large number of studies relating the speed of overarm throw with different anthropometric, technical, or performance variables, to the knowledge of the authors, there are currently no studies referring to the overarm throw speed for beach handball players. Therefore, the aim of this study is to establish the relationships of anthropometric characteristics, hand dimensions, and handgrip strength with overarm ball speed in elite beach handball players.

\section{Material and methods}

\section{Subjects}

Overall, 11 professional beach handball male players (mean age: $26.8 \pm 4.9$ years, range: $20-37$ years; body weight: $84.5 \pm 7.9 \mathrm{~kg}$; body height: $1.85 \pm 6.90 \mathrm{~m}$; training experience: 9.5 years; training work: 24.5 hours per week) of the Spanish national team were recruited for the study. The players took part in the Royal Spanish Handball Federation technical training meeting aimed at preparing for the Beach Handball World Championships held in Budapest, Hungary (2016). The male Spanish team became ranked fifth in this competition and were winners of the European championship tournament in Zagreb, Croatia (2017). No specific exercises for increasing hand strength or specific hand training exercises were applied. All subjects had no pain or disabilities in the upper or lower body.

\section{Procedures}

Data collection was conducted with permission of the Royal Spanish Handball Federation, as well as coaches and players. Before the data collection day, a technical meeting was held to inform players about the tests to be performed. In addition, all participants were informed verbally and in writing about of the risks of the tests and the testing protocol. The test was carried out on the first day of technical training meeting before the Beach Handball World Championships. All measurements of anthropometric parameters were collected during the first day. The field tests were completed in the morning of the second day.

\section{Measurements of anthropometric parameters}

The assessment of the anthropometric parameters followed the protocol of the International Society for the Advancement of Kinanthropometry (ISAK). All players were measured during season control in postprandial state, with light clothing, and barefoot. In addition to basic measurements (height, body mass, and arm span), skeletal breadths were determined with an anthropometer model 101 (GPM, Switzerland) and perimeters were obtained with a Lufkin Executive Thinline tape (Lufkin, W606PM, USA). All anthropometric dimensions were established by an accredited ISAK level 2 anthropometrist, which ensures a protocol with a margin of error $<1 \%$. Weight was measured with $0.1-\mathrm{kg}$ accuracy by means of a Tanita BC- $545 \mathrm{~N}$ balance (Tanita Co., Tokyo, Japan).

\section{Specific hand anthropometric parameters}

Hand anthropometric dimensions measurement followed a specific protocol, which focused on finger spans and lengths and hand polygons [15], as shown in Figure 1. 


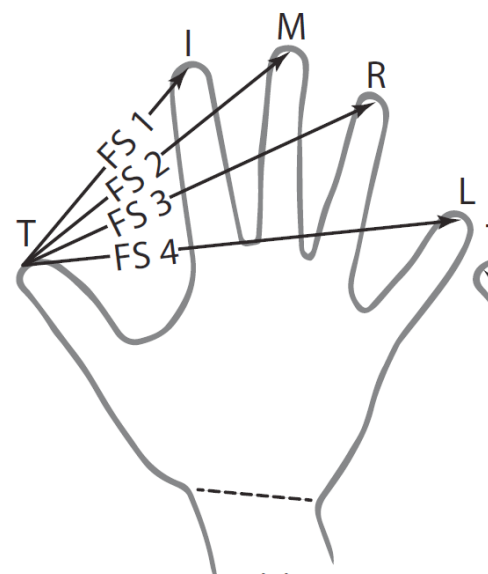

(a)

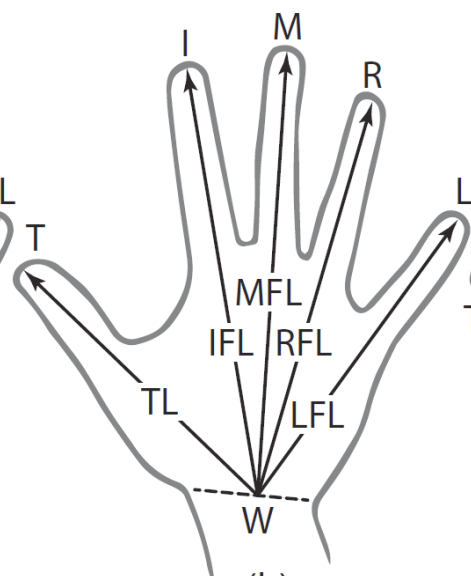

(b)

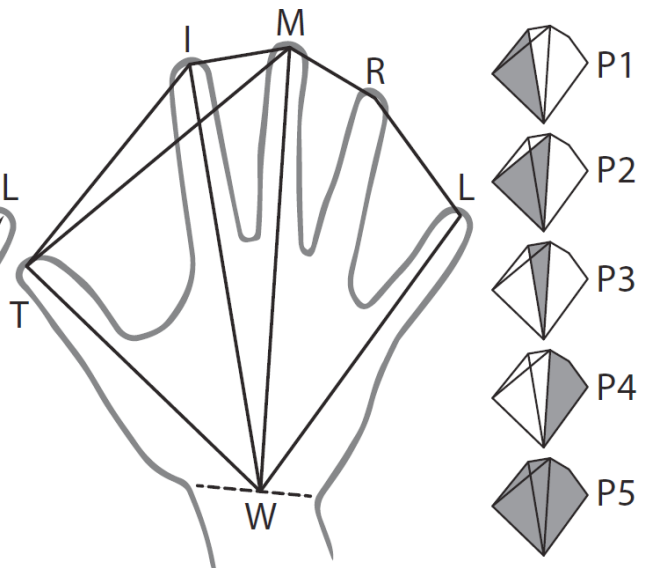

(c)

Figure 1. Fingers spans (a): a) from the tip of the thumb (T) to the tip of the index finger (I), called finger span 1 (FS1); b) from the tip of $\mathrm{T}$ to the tip of the middle finger (M), called finger span 2 (FS2); c) from the tip of T to the tip of the ring

finger (R), called finger span 3 (FS3); d) from the tip of T to the tip of the little finger (L), called finger span 4 (FS4); e) from the tip of $\mathrm{T}$ to the tip of each finger, called finger span 5 (FS5).

Finger lengths (b) were measured between the wrist joint (W) and the tip of the fingers: a) from $\mathrm{W}$ to the tip of T (TL);

b) from W to the tip of I (IFL); c) from W to the tip of M (MFL); d) from W to the tip of R (RFL); e) from W to the tip of $\mathrm{L}(\mathrm{LFL})$.

Hand polygons (c) were measured: a) from W to the tip of T, to the tip of I, and back to W (P1); b) from W to the tip of T, to the tip of M, and to W (P2); c) from W to the tip of I, to the tip of M, and to W (P3); d) from W to the tip of I, to the tip of M, to the tip of R, to the tip of L, and to W (P4); e) from W to the tip of T, to the tip of I, to the tip of M, to the tip of R, to the tip of L, and to W (P5)

Hand dimensions were measured directly by digital evaluation of the handprint drawn on paper by means of a 2-dimensional scanner (Sensor Medica, Rome, Italy) and digitising software (Kinovea, France). Hand measurements were repeated after a week to test for intra-observer reliability by means of the intra-class correlation coefficient (ICC) and coefficient of variation (CV). All measures (see Figure 1 for the explanations of finger spans, finger lengths, and hand polygons) showed proper reliability, expressed as the following ICC and CV values: $0.990,0.51 \%$ for $\mathrm{FS} 1$; $0.990,0.42 \%$ for FS2; $0.999,0.38 \%$ for FS3; 0.998, $0.44 \%$ for FS4; $0.999,0.30 \%$ for FS5; $0.982,0.50 \%$ for TL; 0.986 , $0.47 \%$ for IFL; $0.987,0.37 \%$ for MFL; $0.984,0.41 \%$ for RFL; 0.982, $0.50 \%$ for LFL; 0.996, $0.41 \%$ for P1; $0.996,0.32 \%$ for $\mathrm{P} 2$; $0.995,0.34 \%$ for P3; 0.991, $0.40 \%$ for P4; and $0.997,0.34 \%$ for P5.

\section{Maximal isometric handgrip force test}

Maximal isometric handgrip force was measured with a digital dynamometer (Grip-D T.K.K. 5401, Takei Scientific Instruments Co., Japan), with the accuracy of $0.1 \mathrm{~kg}$ or $10 \mathrm{~N}$. The dominant hand was determined on the basis of the subjects' preference in throwing a ball or in usual activities like holding a pencil or eating. The participants performed the test in a standing position, with the dynamometer set parallel to the body without contact. The position of the hand remained constant in downward direction. The palm and arm did not flex during measurements. The subjects performed two repetitions to warm up, followed by maximal force strength on the dynamometer during 5 seconds. All players completed three trials with 3-minute rest between trials to minimize the effects of fatigue. The mean value of the three trials was used. The dominant hand measurements showed ICC mean value of 0.994 and $\mathrm{CV}$ of $2.22 \%$ between trials.

\section{Throwing velocity test}

The throwing velocity was assessed with a radar gun (Stalker Pro Inc., Texas, USA), set at a recording frequency of $33 \mathrm{~Hz}$ and accuracy of $0.045 \mathrm{~m} \cdot \mathrm{s}^{-1}$. The radar gun was placed behind the goal post in a perpendicular direction to the player. Before each test, players performed a 15-minute warm-up, composed of vegetative activation during 5 minutes and submaximal throws without a goalkeeper for the remaining 10 minutes. Later, they were asked to throw a ball at maximum velocity in 3 different throw types: (a) standing throw at the 6-m line without displacement (ST); (b) standing throw after 2 steps from the 6-m line (RST); (c) jump throw with a run-up and self- 
spin from the 6-m line (RSST). Each throw was performed with no intervention of a goalkeeper. Three throws of each type were performed and the mean values were used for further analysis. A 3-minute rest time between the throws was applied to avoid fatigue. Throw measurements showed good test-retest reliability, as shown by the ICC and CV values: ICC $=0.944$, $\mathrm{CV}=3.5 \%$ for $\mathrm{ST}$; ICC $=0.987, \mathrm{CV}=3.9 \%$ for RST; and $\mathrm{ICC}=0.991, \mathrm{CV}=4.8 \%$ for RSST [18].

\section{Statistical analysis}

Mean and standard deviation values were used for descriptive analysis. The Shapiro-Wilk test was performed to test for normal distribution. Bivariate Pearson's product-moment correlation coefficient $(r)$ with 95\% confidence intervals (CI) via bootstrapping was applied to determine relationships between independent variables. The magnitude of correlation coefficients was interpreted in accordance with the augmented thresholds proposed by Hopkins et al. [19]: $\geq 0.5$ for large, $\geq 0.7$ for very large, and $\geq 0.9$ for extremely large, whereas magnitudes $<0.5$ were considered as trivial given the lack of prediction purposes in practice. The effects of anthropometric variables, hand specific measures and handgrip strength related with the throw test were analysed with stepwise multiple regression analysis. All statistical analyses were performed with the use of SPSS v.22 statistical software (SPSS Inc, Chicago, USA).

Correlation coefficient magnitudes were evaluated qualitatively with magnitude-based inference [19] since $p$-values in null hypothesis significance testing are sample-size dependent and fail to give information about the magnitude of effects. Magnitude-based inference involves $90 \% \mathrm{CI}$, representing the uncertainty in the true value of $r$. The smallest worthwhile change, as the minimum improvement likely to have a practical impact, is expressed in 3 scales: substantially positive, trivial, and substantially negative [20]. Chances that the true values lie on these scales are calculated by comparison with CI: if the chance overlaps positive and negative values substantially, i.e. the true value could be in a positive and a negative sense larger than $5 \%$ simultaneously, then the true value is deemed as unclear. Else it is clear and is estimated to have the magnitude of the observed value with the following probabilistic terms: possibly: 25-75\%; likely: 75-95\%; very likely: 95-99.5\%; and most likely: > 99.5\%. In order to look for meaningful correlations, the smallest worthwhile change for $r$ was set to 0.5. Such mechanistic inferences and confident limits were calculated with an available spreadsheet [21].

\section{Ethical approval}

The research related to human use has been complied with all the relevant national regulations and institutional policies, has followed the tenets of the Declaration of Helsinki, and has been approved by the Ethics Committee of the University of Alicante.

\section{Informed consent}

Informed consent has been obtained from all individuals included in this study.

\section{Results}

The specific hand, upper limb, and body anthropometric measures are presented in Table 1.

The maximal throw speed test results were as follows: $25.64 \pm 4.14 \mathrm{~m} \cdot \mathrm{s}^{-1}$ for ST, $23.71 \pm 4.24 \mathrm{~m} \cdot \mathrm{s}^{-1}$ for RST, and $24.83 \pm 5.49 \mathrm{~m} \cdot \mathrm{s}^{-1}$ for RSST.

Table 1. Descriptive anthropometric measures

\begin{tabular}{|c|c|c|c|}
\hline Variable & Mean & $S D$ & $95 \% \mathrm{CI}$ \\
\hline FS1 (mm) & 124.2 & 24.3 & $110.9-138.00$ \\
\hline $\mathrm{FS} 2(\mathrm{~mm})$ & 168.3 & 26.3 & 153.9-182.6 \\
\hline FS3 (mm) & 190.5 & 28.1 & 176.2-205.9 \\
\hline $\mathrm{FS} 4(\mathrm{~mm})$ & 206.7 & 25.9 & 193.9-221.1 \\
\hline FS5 (mm) & 276.5 & 34.2 & 259.7-295.6 \\
\hline $\mathrm{TL}(\mathrm{mm})$ & 143.8 & 6.2 & $140.2-147.4$ \\
\hline IFL (mm) & 189.1 & 8.5 & 184.3-194.3 \\
\hline MFL (mm) & 195.9 & 8.6 & $191.1-201.3$ \\
\hline RLF (mm) & 185.9 & 8.1 & 181.3-190.7 \\
\hline LFL (mm) & 161.1 & 7.8 & $156.5-165.8$ \\
\hline P1 (mm) & 457.1 & 33.3 & $438.7-476.5$ \\
\hline P2 (mm) & 508.1 & 32.8 & $489.8-526.8$ \\
\hline P3 (mm) & 439.1 & 18.5 & $428.0-449.4$ \\
\hline $\mathrm{P} 4$ (mm) & 544.3 & 25.6 & $441.1-471.2$ \\
\hline P5 (mm) & 581.4 & 41.9 & 449.8-606.3 \\
\hline Height (cm) & 185.5 & 8.3 & 180.5-190.6 \\
\hline Body mass (kg) & 84.4 & 8.4 & $79.7-89.0$ \\
\hline Arm span (cm) & 189.0 & 7.53 & 184.7-193.4 \\
\hline BMI $\left(\mathrm{kg} \cdot \mathrm{m}^{-2}\right)$ & 24.6 & 1.5 & $23.8-25.4$ \\
\hline DH (kg) & 50.8 & 4.1 & $48.5-53.1$ \\
\hline $\mathrm{NDH}(\mathrm{kg})$ & 48.7 & 5.7 & $45.9-52.1$ \\
\hline $\mathrm{AP}(\mathrm{cm})$ & 34.1 & 2.5 & $32.5-35.4$ \\
\hline $\mathrm{CAP}(\mathrm{cm})$ & 36.6 & 2.5 & $35.1-37.8$ \\
\hline $\operatorname{EED}(\mathrm{cm})$ & 7.2 & 0.6 & $6.9-7.6$ \\
\hline EDW (cm) & 5.8 & 0.7 & $5.5-6.3$ \\
\hline
\end{tabular}

See Figure 1 for the explanations of finger spans, finger lengths, and hand polygons. BMI - body mass index, $\mathrm{DH}$ - strength of dominant hand, NDH - strength of non-dominant hand, AP - arm perimeter, CAP - contracted arm perimeter, EED - elbow epicondyle distance, EDW - epicondyle distance from wrist 


\section{HUMAN MOVEMENT}

J.M. Jimenez-Olmedo et al., Anthropometric parameters and overarm throw

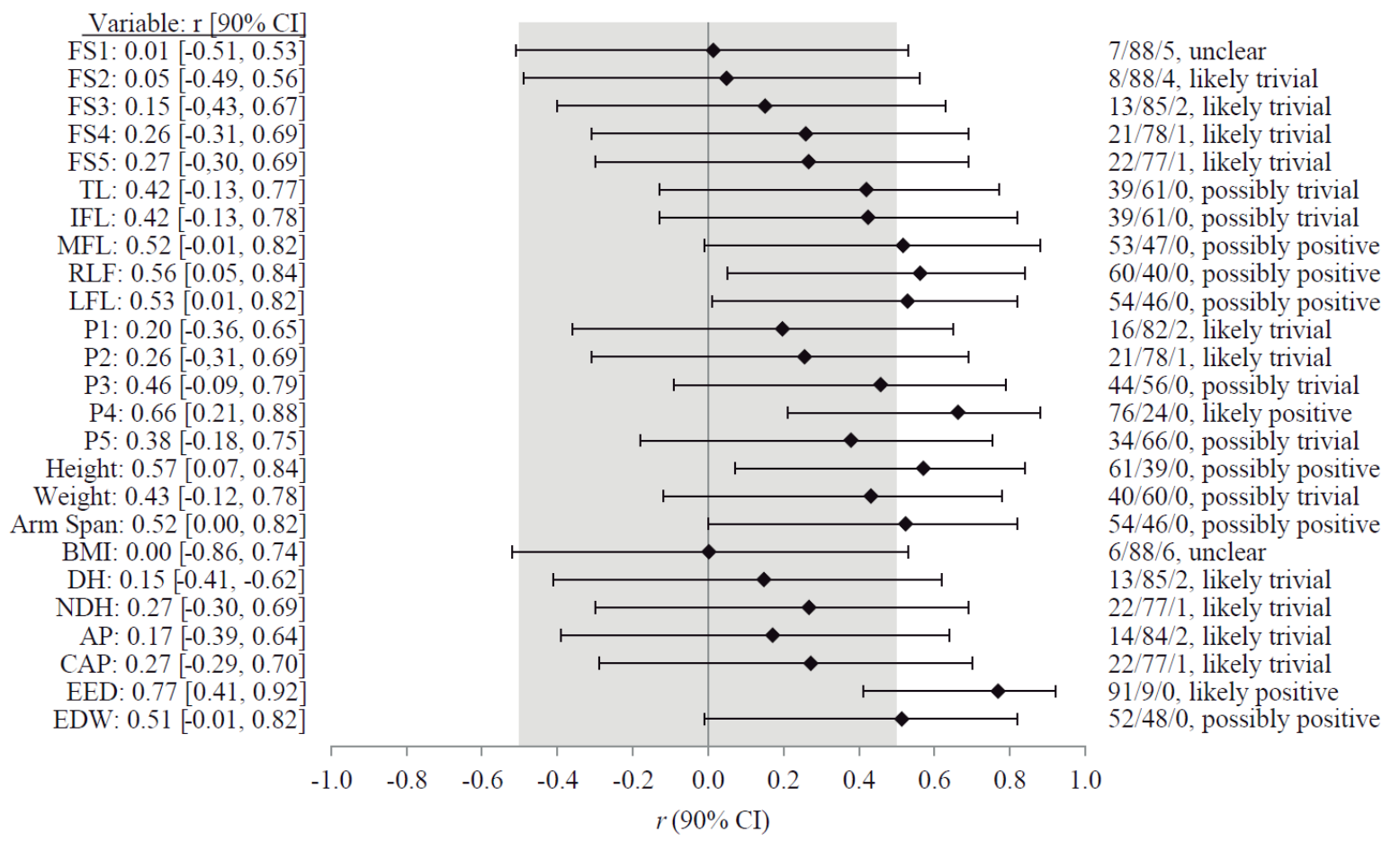

Figure 2. Correlations between maximal standing throw speed and the anthropometric measures. On the left, values are presented as $r$ magnitudes with $90 \%$ confidence intervals for each anthropometric variable. Shaded area denotes the smallest worthwhile change representing trivial outcomes. On the right, qualitative probabilistic mechanistic inference

about the true effect is displayed, together with the percentage probabilities for the correlation to be substantially positive / trivial / substantially negative. See Figure 1 and Table 1 for the explanations of the anthropometric measures

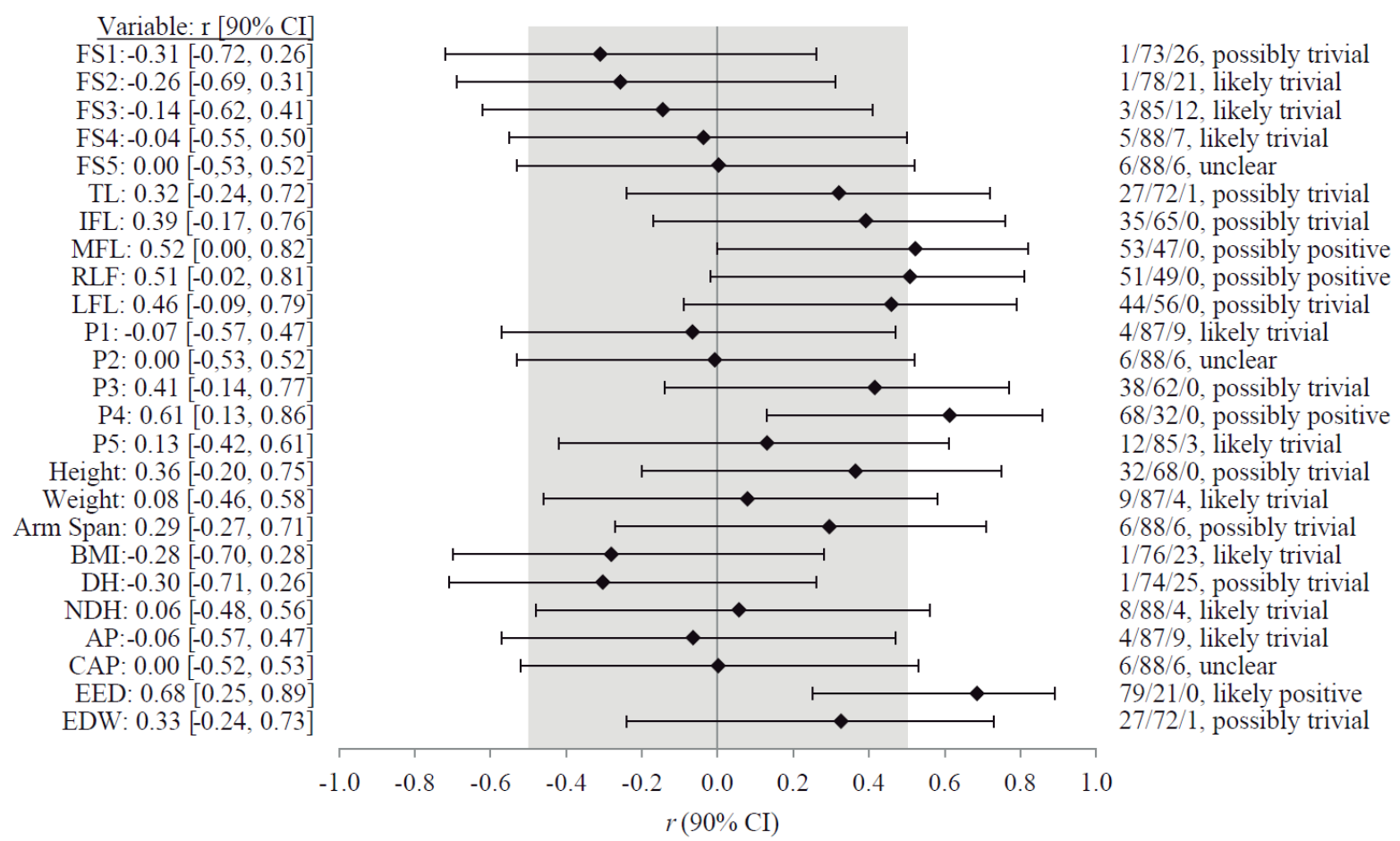

Figure 3. Correlations between maximal standing throw after two steps speed and the anthropometric measures. On the left, values are presented as $r$ magnitudes with $90 \%$ confidence intervals for each anthropometric variable. Shaded area denotes the smallest worthwhile change representing trivial outcomes. On the right, qualitative probabilistic mechanistic inference about the true effect is displayed, together with the percentage probabilities for the correlation to be substantially positive / trivial / substantially negative. See Figure 1 and Table 1 for the explanations of the anthropometric measures 


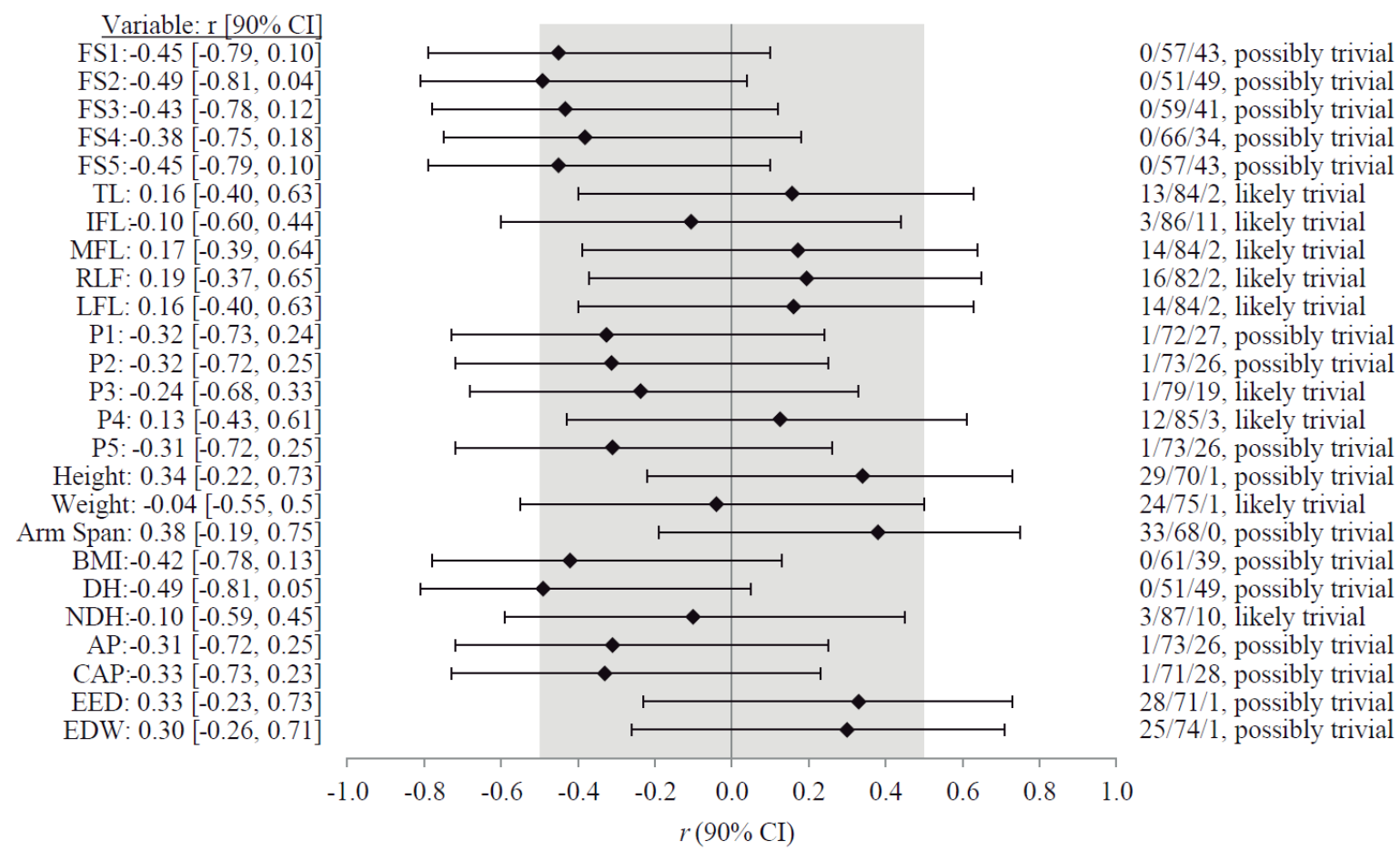

Figure 4. Correlations between maximal jump throw with a run-up and self-spin speed and the anthropometric measures. On the left, values are presented as $r$ magnitudes with $90 \%$ confidence intervals for each anthropometric variable. Shaded area denotes the smallest worthwhile change representing trivial outcomes. On the right, qualitative probabilistic mechanistic inference about the true effect is displayed, together with the percentage probabilities for the correlation to be substantially positive / trivial / substantially negative. See Figure 1 and Table 1 for the explanations of the anthropometric measures

The correlations between anthropometric measures and maximal speed for the ST, RST, and RSST are presented together with the qualitative assessment and percentage probabilities to be substantially positive, trivial, and substantially negative. The largest magnitudes of correlations with ST were found for the following anthropometric measures (Figure 2): elbow epicondyle distance (EED) ( $r=0.77$, likely positive), P4 ( $r=0.66$, likely positive), height ( $r=0.57$, possibly positive), RFL ( $r=0.56$, possibly positive), LFL ( $r=0.53$, possibly positive), MFL ( $r=0.52$, possibly positive), and arm span ( $r=0.52$, possibly positive). These correlations are considered as large, except for that regarding EED, which was very large.

RST showed similar largest magnitudes of correlations (Figure 3): EED ( $r=0.68$, likely positive), P4 ( $r=0.61$, possibly positive), RFL $(r=0.51$, possibly positive), and MFL ( $r=0.52$, possibly positive). These correlations are considered as large.

Finally, all correlations for RSST resulted in magnitudes below 0.5 and qualitative assessments were possibly and likely trivial (Figure 4).

Stepwise multiple regression analysis of hand and upper limb anthropometric measures as independent variables established the following prediction relationships with the throw speed test: ST was predicted by P4 in $44 \%\left(R^{2} \times 100\right)$, RST was predicted by $\mathrm{P} 4$ in $37 \%$ $\left(R^{2} \times 100\right)$ and by combining P4 and FS1 in $68 \%\left(R^{2} \times\right.$ $100)$, ST was predicted by humerus diameter in $59 \%$ $\left(R^{2} \times 100\right)$, and RST was predicted by humerus diameter in $47 \%\left(R^{2} \times 100\right)$.

\section{Discussion}

The purpose of the study was to explore possible relationships between anthropometric measures, hand dimensions, and handgrip strength with overarm ball speed in elite beach handball players.

It is worth mentioning that literature is focused on studies referring to throws in team handball, but studies on beach handball are scarce. Therefore, the comparison between outcomes in throwing tests registered in both disciplines, as well as the comparison of anthropometrics parameters can help to understand differences between these two sports.

Furthermore, beach handball players show lower height and weight values than team handball players of similar level [22]. Also, beach handball players are 
J.M. Jimenez-Olmedo et al., Anthropometric parameters and overarm throw

around 6\% lighter and around 2\% shorter than indoor players. These characteristics could be related to the kinematics of playing on a different surface like sand. Therefore, specific pattern movements need to be developed to optimize game performance [23]. A similar trend can be found in other sand sports, such as beach volleyball, where defenders are lighter and shorter but also more agile and faster than blockers [24]. Accordingly, beach handball players need to be lighter and shorter than indoor handball players to optimize movement on soft surfaces.

Other anthropometric measurements explored in different studies were the diameters of wrist and elbow joints. These showed similar values in beach handball players and handball indoor players [13]. This is an important part of overarm throw as the hand and wrist segments present the maximal endpoint velocity followed by elbow and shoulder [6]. It is worth to highlight that the wrist plays a role in throw accuracy since it is the last joint that players can operate to change the ball direction, together with elbow.

In beach and team handball, throw performance is influenced by the interaction of a number of anthropometric variables. To the knowledge of the authors, this study is the first to show a very large correlation $(r=0.77,90 \%$ CI: 0.41-0.92) between maximal ST speed and the EED measure. According to the mechanistic inference (magnitude-based inference), this result is likely positive, with a $90.7 \%$ chance that the true value of the effect statistics is substantially positive and $0.0 \%$ negative. Similarly, maximal RST throw speed shows large correlation with EED $(r=0.68,90 \%$ CI: 0.25-0.89), which is a likely positive result, with a $79.3 \%$ chance that the true value of the effect statistics is substantially positive and $0.0 \%$ negative. Among indoor players, elbow does not affect technical actions, but only throw velocity. Since similar ball speed values are registered in beach and team handball throw tests, similar conditional throw factors can be established for both disciplines [6]. In team handball, the ball speed is determined by the position of arms. When arms are extended backwards, the ball speed is significantly different than that with arms parallel to hip or in horizontal position [25]. Other studies have established that rotational strength of shoulder is not related to ball speed [26], but the function of shoulder and elbow is basically to stabilise and direct the position of elbow. The latter could be bound with ball stabilization since rotation axes changes associated with the use of interaction torque during the acceleration phase could be a determinant factor in the production of hand velocity at ball release [27]. Such a ball release mechanism could explain the correlation between elbow diameter and values registered in throw tests.

On the other hand, with regard to hand anthropometric measures, studies have shown relationships between hand dimensions and throw performance [14]. However, hand dimensions alone cannot predict ball speed in throw tests. Therefore, basic anthropometric parameters are more important than hand dimensions in throw speed because overarm throw is a multi-joint movement, with many potential degrees of freedom.

The presented study has shown large correlations between the P4 hand measure and ball speed for both ST $(r=0.66,90 \%$ CI: $0.21-0.88)$ and RST $(r=0.61$, $90 \%$ CI: 0.13-0.86), being likely and possibly positive, respectively. This result is also supported by possibly large correlations shown for the middle, ring, and little finger lengths (MFL, RFL, and LFL, respectively), which compose the $\mathrm{P} 4$ polygon. Also, ST and RST are predicted by $\mathrm{P} 4$ in $44 \%$ and $37 \%$, respectively. This aspect can be explained by ball dimensions, weight, and texture, different than those in indoor handball, which may influence throw kinematics, as reflected in the overarm throw in different sports [28].

Regarding dimensions and force exerted by hands, beach handball players presented lower handgrip strength and lower values in finger spans and finger perimeters than athletes tested in previous studies [29]. Also, positive correlations with handgrip strength and hand dimensions for athletes only were found. In this study, hand strength showed no positive correlation with hand size, the exerted force, or ball speed. Therefore, it is possible that hand strength might not be crucial in beach handball since the ball texture allows players to hold it with little force. As compared with indoor players, beach handball players have smaller hands, which could also explain the above lack of correlation.

Finally, in relation to throw tests, ST outcomes in beach handball players were better than in other studies conducted among team handball players; in turn, for RST, indoor players showed higher maximal speed than beach players [11]. Since RSST is a specific overarm throw that includes run-up and self-spin, it cannot be compared with indoor handball. To the knowledge of the authors, there are no studies focused on analysing such a specific throw in beach handball. However, since the remainder of throw types are similar for both disciplines, it would be of interest to establish relationships in overarm throw between sand and indoor surfaces. 


\section{Conclusion}

In this paper, general and hand-specific anthropometric characteristics and hand strength have been correlated with throw performance. The results demonstrated a positive relationship between $\mathrm{P} 4$ hand dimensions and two specific throw tests: ST and RSST. Furthermore, $44 \%$ of throw speed can be predicted with $\mathrm{P} 4$ alone. Elbow perimeters have also shown a positive relationship with ST and RSST performance as elbows play a role in stabilizing throw direction. Contrary to team handball, hand strength is not a factor in throw ball speed, which could be due to a smaller hand size in beach handball players. Therefore, these variables should be taken into account when selecting specialist athletes in beach handball.

\section{Acknowledgments}

The authors would like to thank the Royal Spanish Handball Federation, especially the beach handball technical team: Juan Carlos Zapardiel, Jaime Osborne, and Daniel Lara.

\section{Disclosure statement}

No author has any financial interest or received any financial benefit from this research.

\section{Conflict of interest}

The authors state no conflict of interest.

\section{References}

1. Van den Tillaar R, Marques M. Effect of training on the kinematics and performance in overarm throwing in experienced female handball players. Port J Sport Sci. 2011;11(Suppl. 2):125-128.

2. Van den Tillaar R, Ettema G. Influence of instruction on velocity and accuracy of overarm throwing. Percept Mot Skills. 2003;96(2):423-434; doi: 10.2466/PMS. 96.2.423-434.

3. Van den Tilaar R, Ettema G. Instructions emphasizing velocity, accuracy, or both in performance and kinematics of overarm throwing by experienced team handball players. Percept Mot Skills. 2003;97(3 Pt 1): 731-742; doi: 10.2466/PMS.97.7.731-742.

4. Debanne T, Laffaye G. Predicting the throwing velocity of the ball in handball with anthropometric variables and isotonic tests. J Sports Sci. 2011;29(7):705713; doi: 10.1080/02640414.2011.552112.

5. Marques MC, van den Tilaar R, Vescovi JD, Gonzalez-Badillo JJ. Relationship between throwing velocity, muscle power, and bar velocity during bench press in elite handball players. Int J Sports Physiol Perform. 2007;2(4):414-422; doi: 10.1123/ijspp.2.4.414.

6. Van den Tillaar R, Cabri JMH. Gender differences in the kinematics and ball velocity of overarm throwing in elite team handball players. J Sports Sci. 2012;30(8): 807-813; doi: 10.1080/02640414.2012.671529.

7. Andrade MS, de Carvalho Koffes F, Benedito-Silva AA, da Silva AC, de Lira CAB. Effect of fatigue caused by a simulated handball game on ball throwing velocity, shoulder muscle strength and balance ratio: a prospective study. BMC Sports Sci Med Rehabil. 2016;8(1):13; doi: 10.1186/s13102-016-0038-9.

8. Wagner H, Buchecker M, von Duvillard SP, Müller E. Kinematic comparison of team handball throwing with two different arm positions. Int J Sports Physiol Perform. 2010;5(4):469-483; doi: 10.1123/ijspp.5.4.469.

9. Wagner H, Pfusterschmied J, von Duvillard SP, Müller E. Skill-dependent proximal-to-distal sequence in team-handball throwing. J Sports Sci. 2012;30(1):2129; doi: 10.1080/02640414.2011.617773.

10. Vila H, Manchado C, Rodriguez N, Abraldes JA, Alcaraz PE, Ferragut C. Anthropometric profile, vertical jump, and throwing velocity in elite female handball players by playing positions. J strength Cond Res. 2012; 26(8):2146-2155; doi:10.1519/JSC.0b013e31823b0a46.

11. Chaouachi A, Brughelli M, Levin G, Boudhina NB, Cronin J, Chamari K. Anthropometric, physiological and performance characteristics of elite team-handball players. J Sports Sci. 2009;27(2):151-157; doi: 10.1080/02640410802448731.

12. Visnapuu M, Jürimäe T. Relations of anthropometric parameters with scores on basic and specific motor tasks in young handball players. Percept Mot Skills. 2009; 108(3):670-676; doi: 10.2466/PMS.108.3.670-676.

13. Srhoj V, Rogulj N, Papić V, Foretić N, Cavala M. The influence of anthropological features on ball flight speed in handball. Coll Antropol. 2012;36(3):967-972.

14. Visnapuu M, Jürimäe T. The influence of basic body and hand anthropometry on the results of different throwing tests in young handball and basketball players. Anthropol Anz. 2008;66(2):225-236.

15. Visnapuu M, Jürimäe T. Handgrip strength and hand dimensions in young handball and basketball players. J Strength Cond Res. 2007;21(3):923-929; doi: 10.1519/ 1533-4287(2007)21[923:HSAHDI]2.0.CO;2.

16. Kosif R, Diramali M. Comparison of all hand digit length ratios in left- and right-handed individuals. Turk J Med Sci. 2012;42(3):545-552; doi: 10.3906/ sag-1006-858.

17. Jimenez-Olmedo JM, Pueo B, Penichet-Tomás A, Chinchilla-Mira JJ, Perez-Turpin JA. Physiological work areas in professional beach volleyball: a case study. Retos: nuevas tendencias en educación física, deporte y recreación. 2017;31:94-97.

18. Manchado C, García-Ruiz J, Cortell-Tormo JM, TortosaMartínez J. Effect of core training on male handball players' throwing velocity. J Hum Kinet. 2017;56(1): 177-185; doi: 10.1515/hukin-2017-0035.

19. Hopkins WG, Marshall SW, Batterham AM, Hanin J. Progressive statistics for studies in sports medicine 


\section{HUMAN MOVEMENT}

J.M. Jimenez-Olmedo et al., Anthropometric parameters and overarm throw

and exercise science. Med Sci Sports Exerc. 2009; 41(1):3-13; doi: 10.1249/MSS.0b013e31818cb278.

20. Buchheit M. The numbers will love you back in return - I promise. Int J Sports Physiol Perform. 2016;11(4): 551-554; doi: 10.1123/IJSPP.2016-0214.

21. Hopkins WG. A spreadsheet for deriving a confidence interval, mechanistic inference and clinical inference from a p value. Sportscience. 2007;11:16-20.

22. Çetin E, Balcı N. The effects of isokinetic performance on accurate throwing in team handball. Procedia Soc Behav Sci. 2015;174:1872-1877; doi: 10.1016/j.sbspro. 2015.01.850.

23. Jimenez-Olmedo JM, Pueo B, Penichet-Tomás A. Defensive systems during the Men's European University Beach Volleyball Championship. J Phys Educ Sport. 2016;16(3):945-950; doi: 10.7752/jpes.2016.03149.

24. Palao JM, Gutiérrez D, Frideres JE. Height, weight, body mass index, and age in beach volleyball players in relation to level and position. J Sports Med Phys Fitness. 2008;48(4):466-471.

25. Van den Tillaar R, Ettema G. Effect of body size and gender in overarm throwing performance. Eur J Appl Physiol. 2004;91(4):413-418; doi: 10.1007/s00421003-1019-8.

26. Zapartidis I, Gouvali M, Bayios I, Boudolos K. Throwing effectiveness and rotational strength of the shoulder in team handball. J Sports Med Phys Fitness. 2007; 47(2):169-178.

27. Hansen C, Rezzoug N, Gorce P, Venture G, Isableu B. Sequence-dependent rotation axis changes and interaction torque use in overarm throwing. J Sports Sci. 2016;34(9):848-885; doi: 10.1080/02640414.2015. 1076167.

28. Van den Tillaar R. The biomechanics of the elbow in overarm throwing sports. Int Sportmed J. 2005;6(1):7-24.

29. Fallahi AA, Jadidian AA. The effect of hand dimensions, hand shape and some anthropometric characteristics on handgrip strength in male grip athletes and non-athletes. J Hum Kinet. 2011;29(1):151-159; doi: 10.2478/v10078-011-0049-2. 